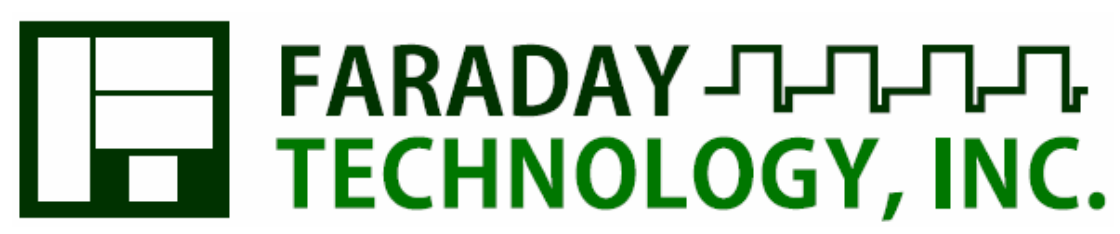

\title{
Multiphysics Modeling Prediction of Electrochemically Machined Through-Hole Geometries
}

\author{
AiMES 2018 \\ Cancún, MX \\ 2 Oct 2018
}

Brian Skinn ${ }^{a}$, Timothy Halla, Stephen Snydera, K.P. Rajurkarb, E.J. Taylor ${ }^{\mathrm{a}}$

a Faraday Technology, Inc., Englewood, OH 45315

${ }^{b}$ Dept. of Mechanical \& Materials Engineering, University of Nebraska - Lincoln, Lincoln, NE 68588 


\section{Electrochemical Machining}

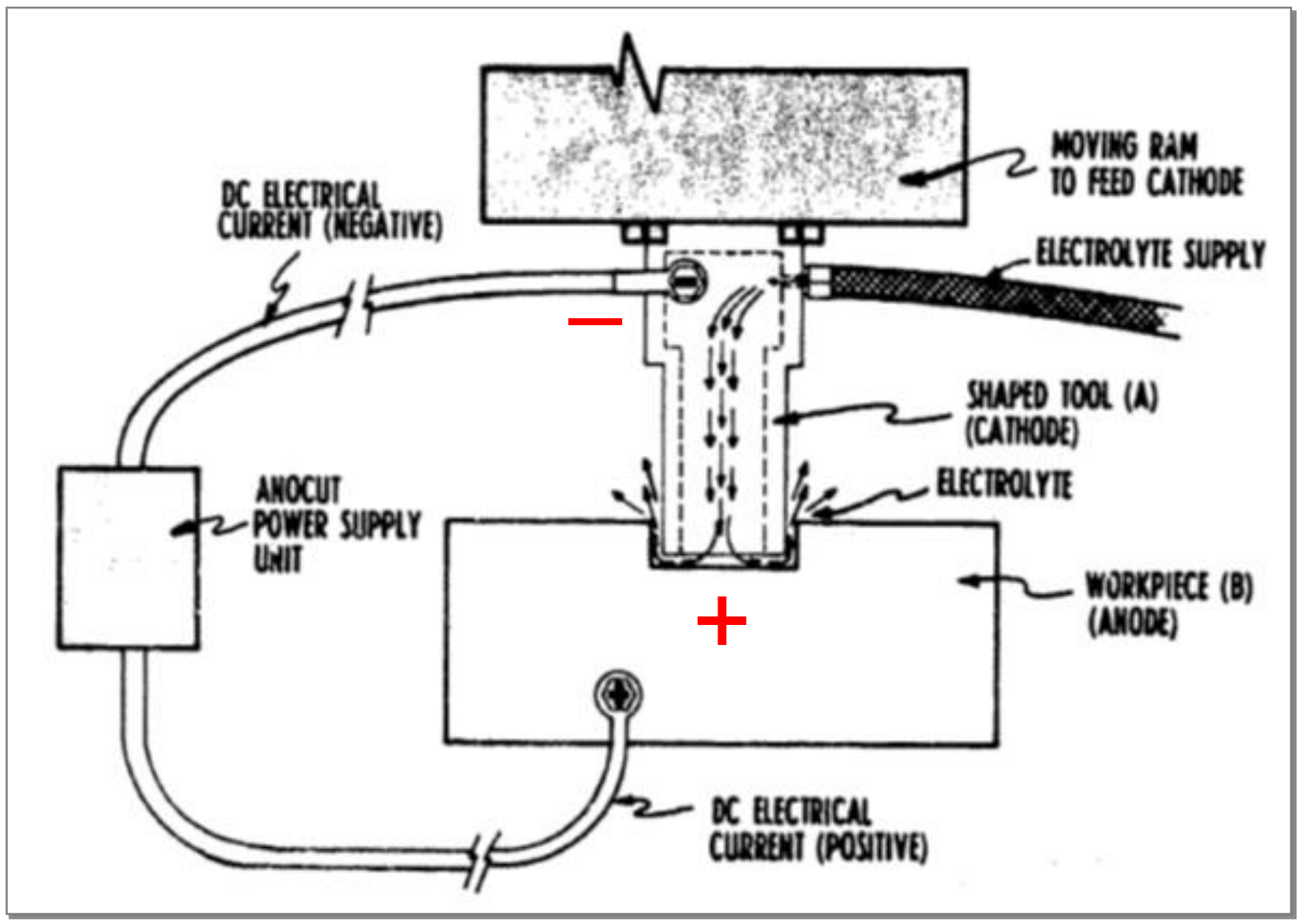

FARADAY ת ת TECHNOLOGY, INC. 


\section{Electrochemical Machining}

Key advantagest:

- Applicability to difficult to cut materials,

- No tool wear/No (thermal/mechanical) surface damage,

- High material removal rate,

- Smooth bright surface finish, and

○ Production of parts with complex geometry.

\section{Key challenges ${ }^{\dagger}:$}

1. Disposal of machining products $\leftarrow$ (prior Faraday)

2. Electrolyte processing $\leftarrow$ (prior Faraday)

3. Tool design $\leftarrow$ (current Faraday)

4. Machining accuracy $\leftarrow$ (current Faraday)

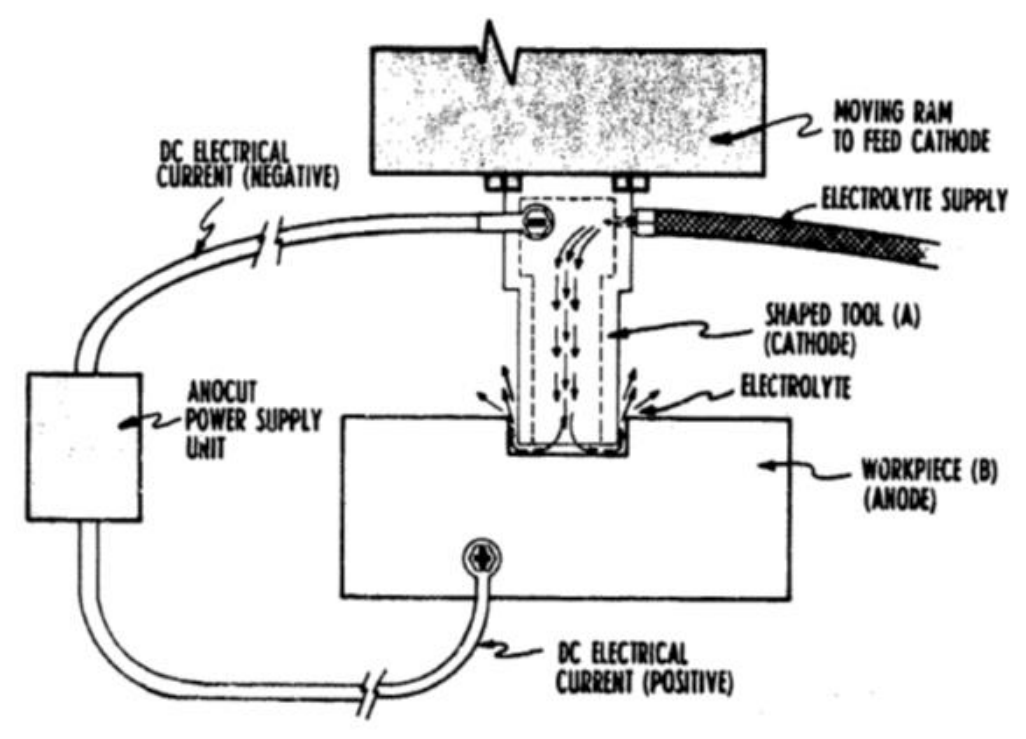

5. Process monitoring $\leftarrow$ (prior sensor/automation advancements)

${ }^{\dagger}$ K.P. Rajurkar, D. Zhu, J.A. McGeough, J. Kozak, A. De Silva, "New Developments in ElectroChemical Machining" Annals of the CIRP Vol 82(2) 1999. 


\section{Target ECM Application: Integrated Muzzle Brakes}

Modular Brake

(from patent literature)

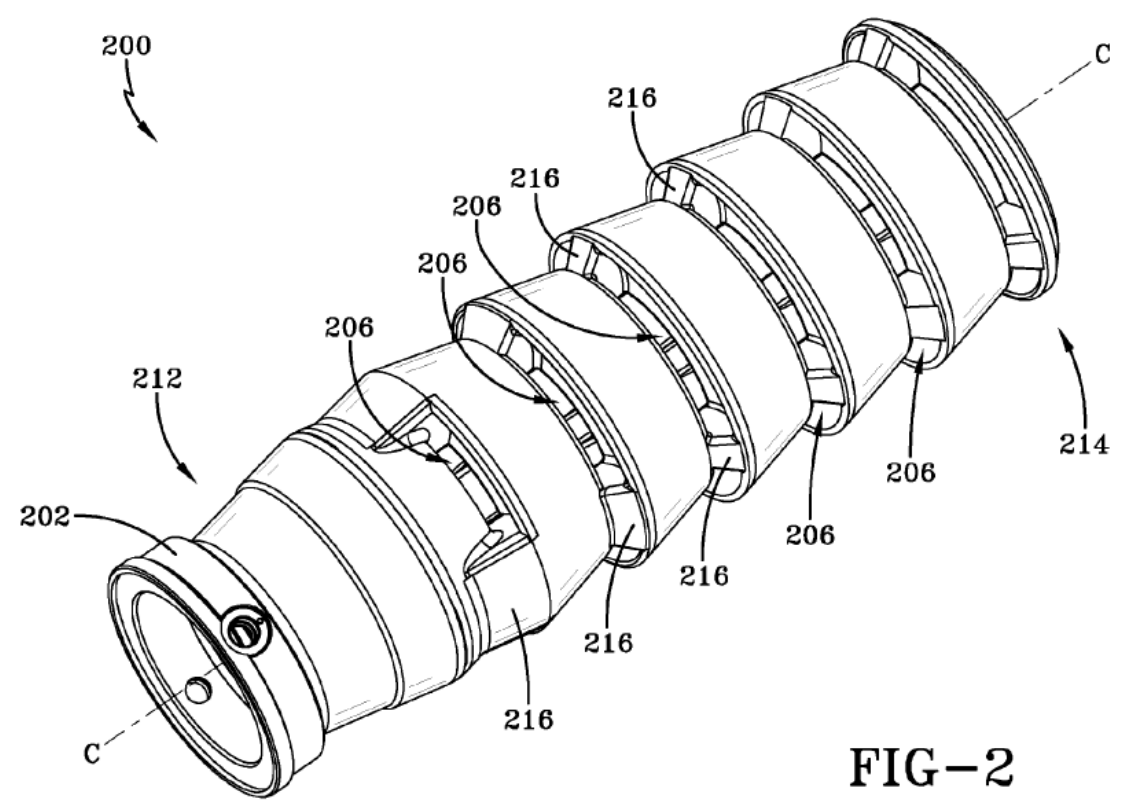

Integrated Brake

(simplified proof-of-concept model)

Cler, D.L. and D. Forliti. U.S. Patent No.

7,600,461, issued 13 Oct 2009

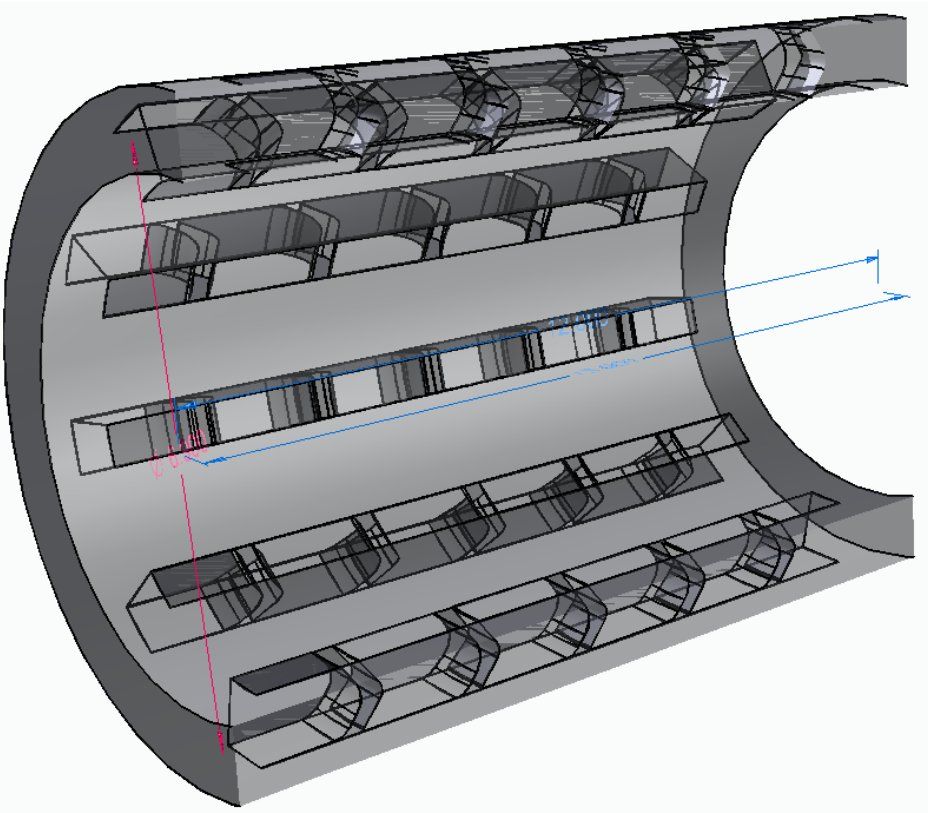

FARADAY תת ת TECHNOLOGY, INC. 


\section{Target ECM Application: Integrated Muzzle Brakes}

Modular Brake

(from patent literature)

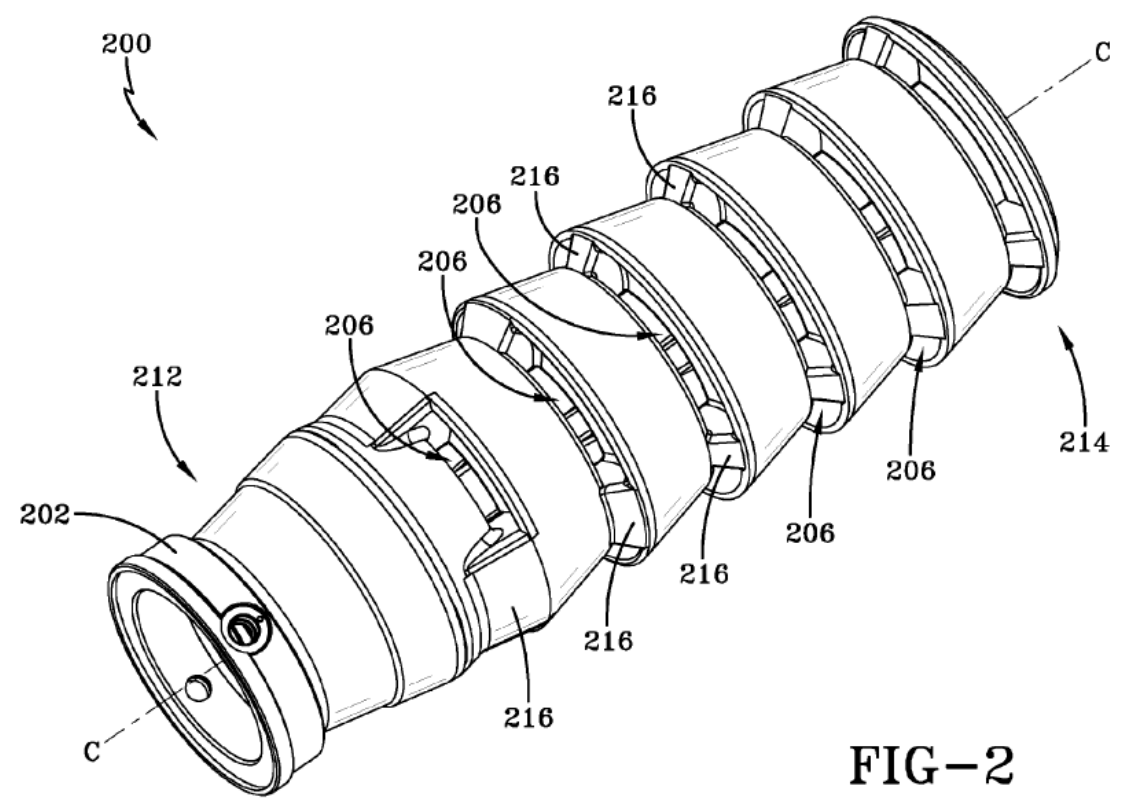

Cler, D.L. and D. Forliti. U.S. Patent No. 7,600,461, issued 13 Oct 2009
Integrated Brake

(simplified proof-of-concept model)

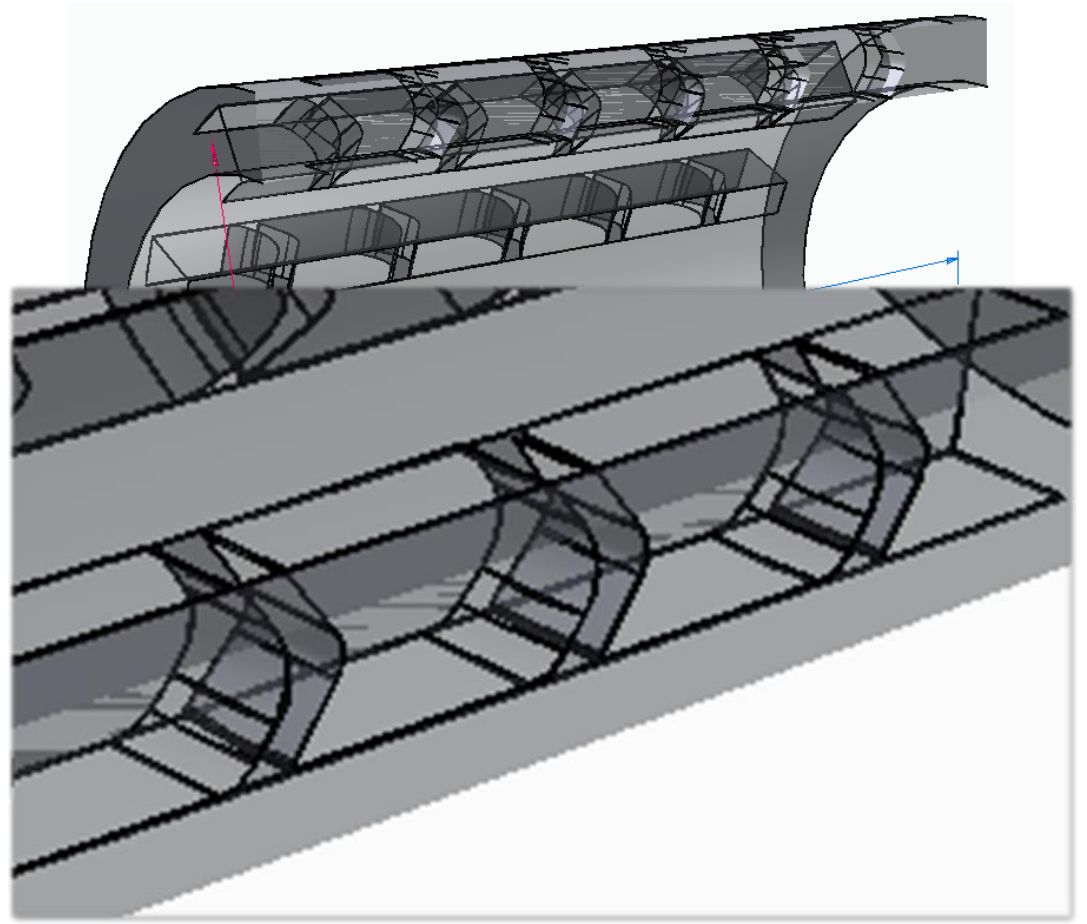

FARADAY תת ת TECHNOLOGY, INC. 


\section{Target ECM Application: Development Strategy}

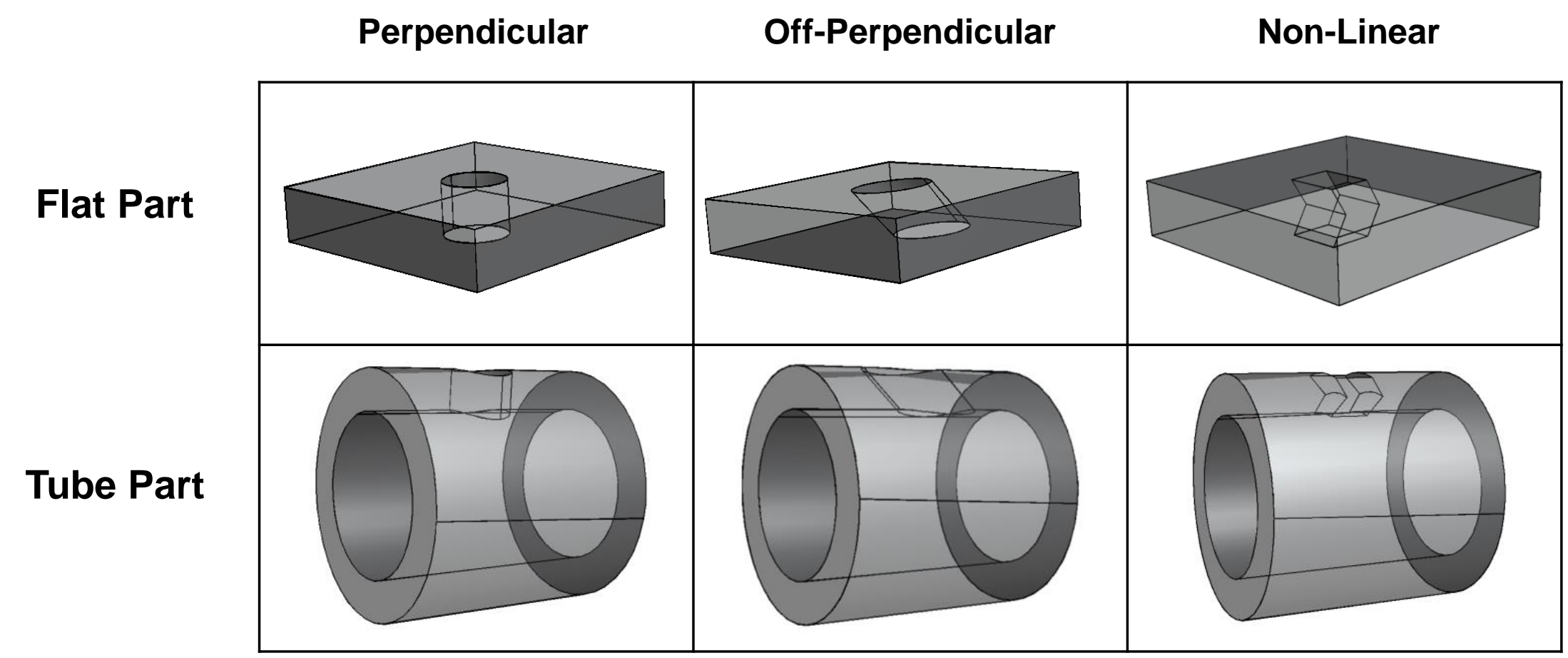

FARADAY תחת TECHNOLOGY, INC. 


\section{FARADAY תЛתЛ,
TECHNOLOGY, INC.}

\section{COMSOL Modeling}

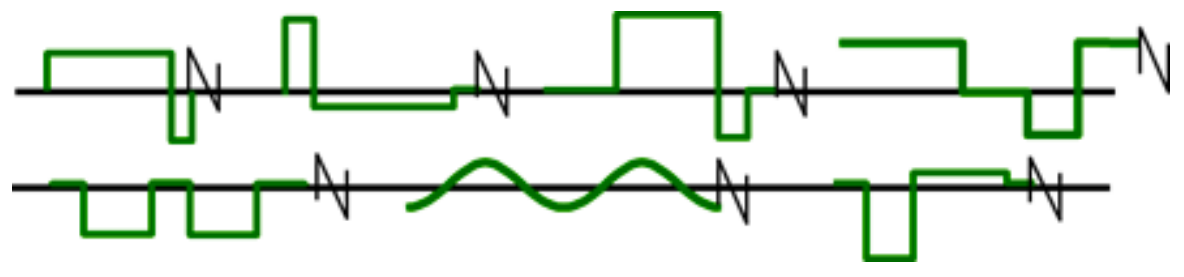




\section{Relevant Physical Phenomena}
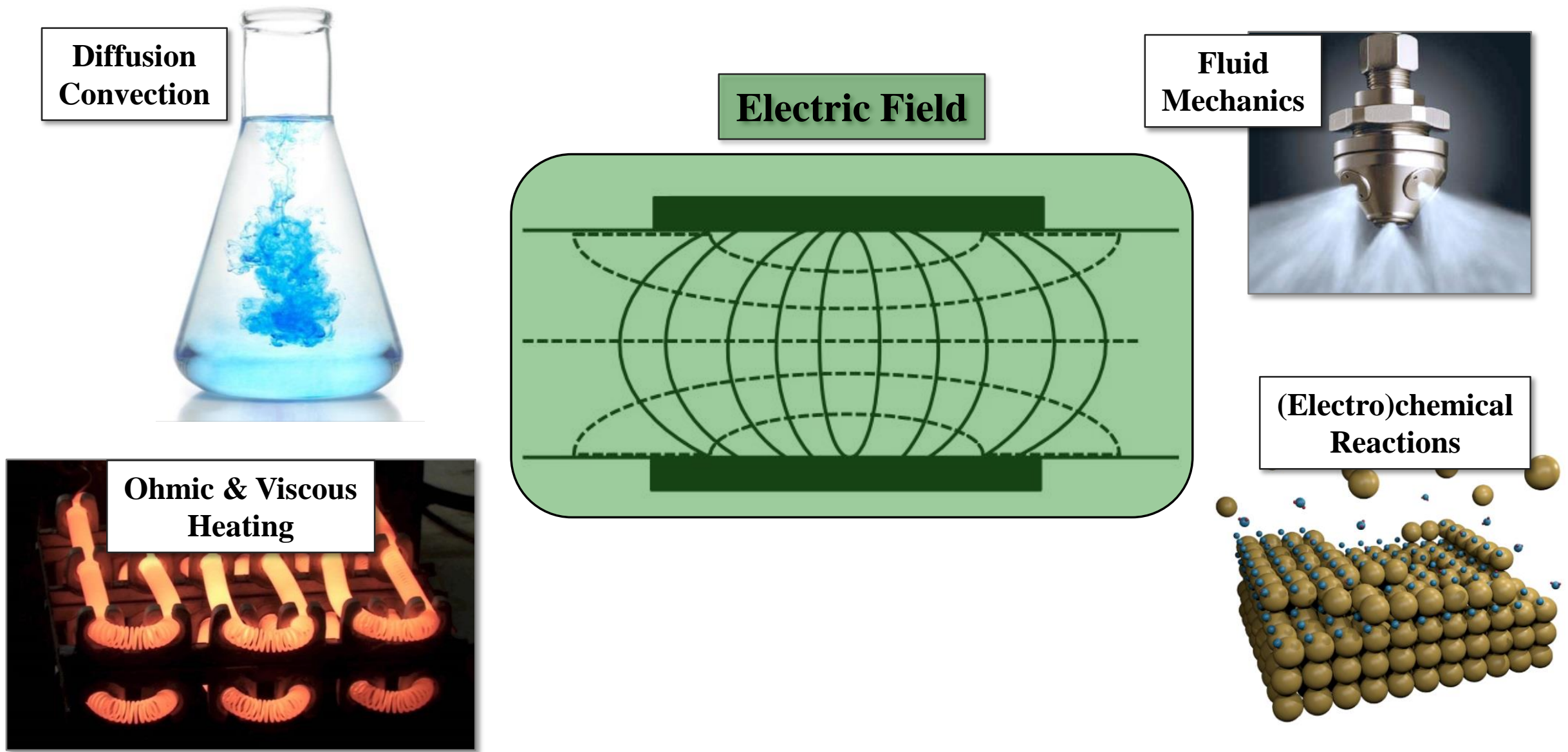

FARADAY ת几Л TECHNOLOGY, INC.

F. Klocke, M. Zeis, S. Harst, A. Klink, D. Veselovac, M. Baumgartner "Modeling and Simulation of the Electrochemical Machining Material Removal Process for the Manufacture of Aero Engine Components" Procedia CIRP 8 265-270 (2013). 


\section{Simulated Physical Phenomena}

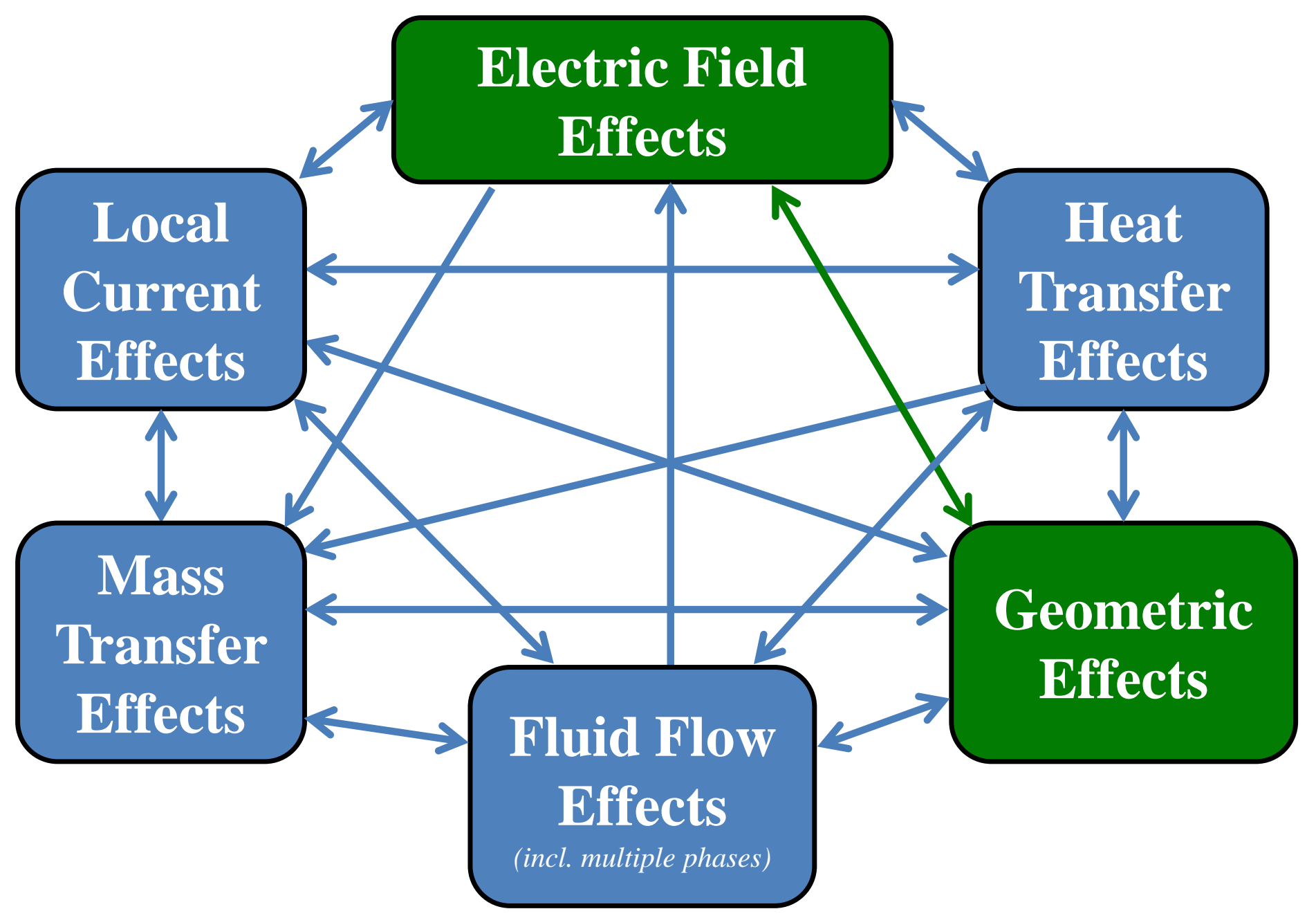

FARADAY ЛПЛ TECHNOLOGY, INC. 


\section{ECM Tool/Flow Configurations}

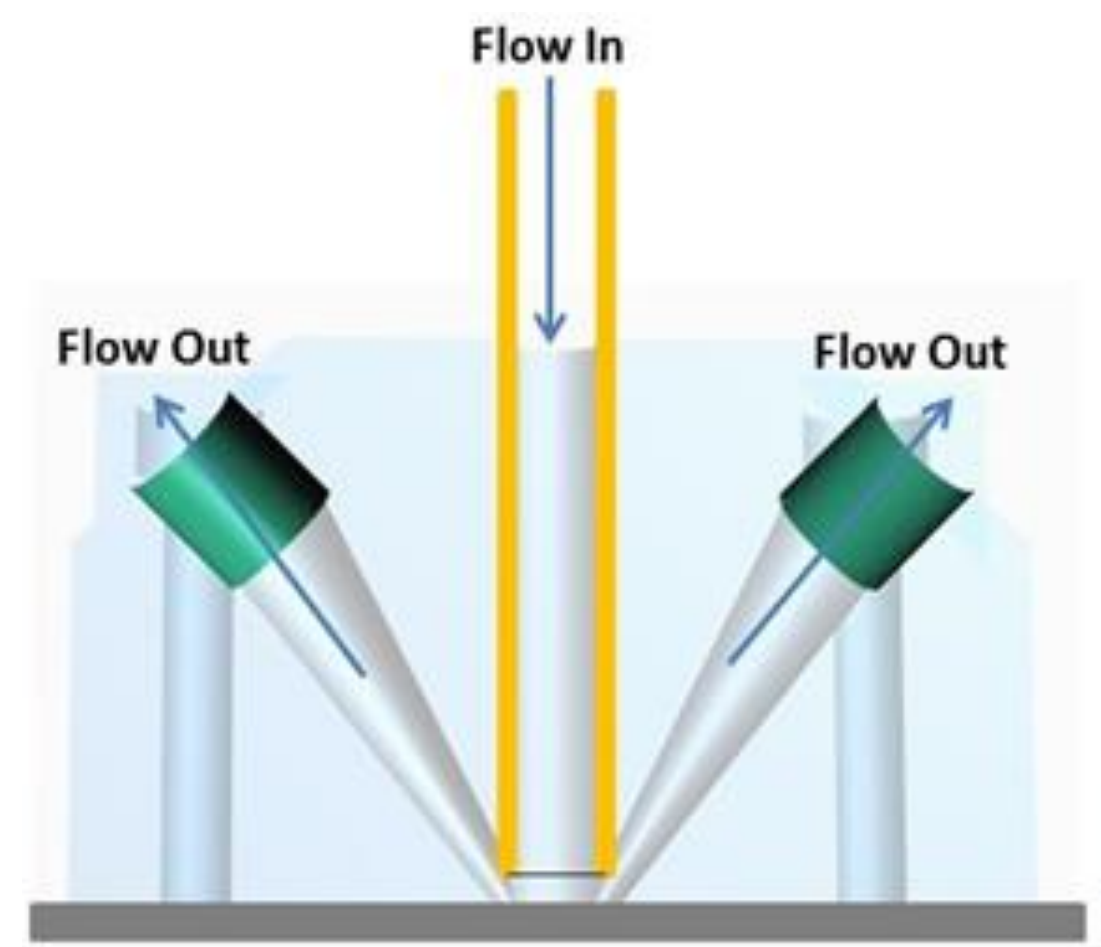

Flow-Through

Tube Tool

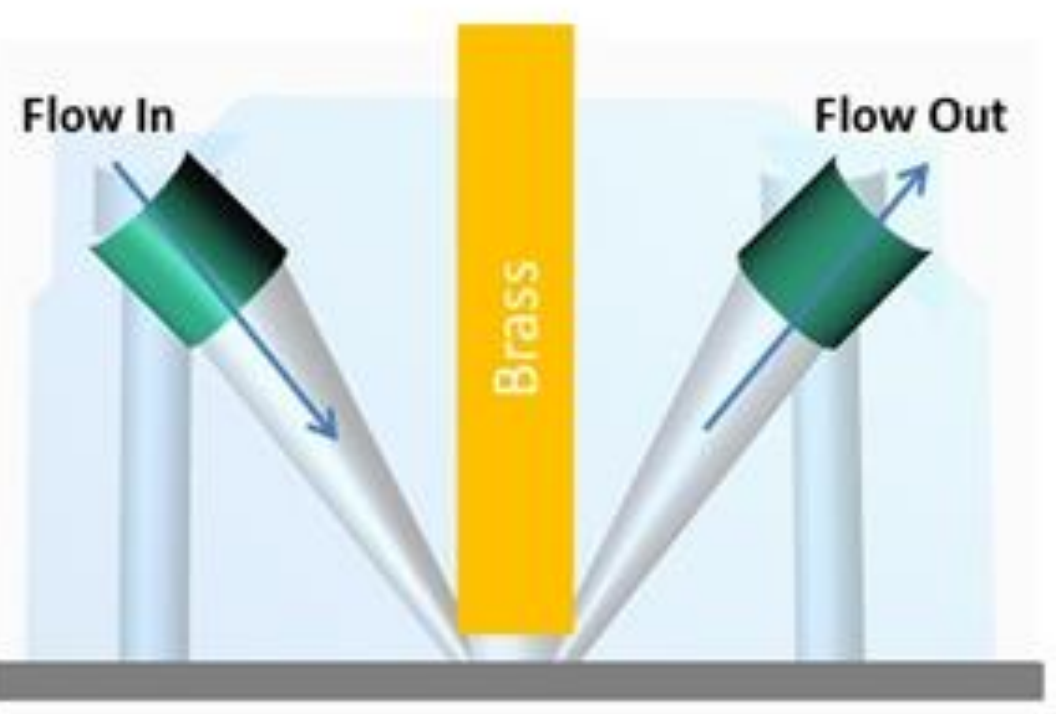

Cross-Flow

Rod Tool

\section{FARADAY תתףת}

TECHNOLOGY, INC. 


\section{Electric Field - Tube vs Rod Tool}

Flow-Through

Tube Tool
Cross-Flow

Rod Tool

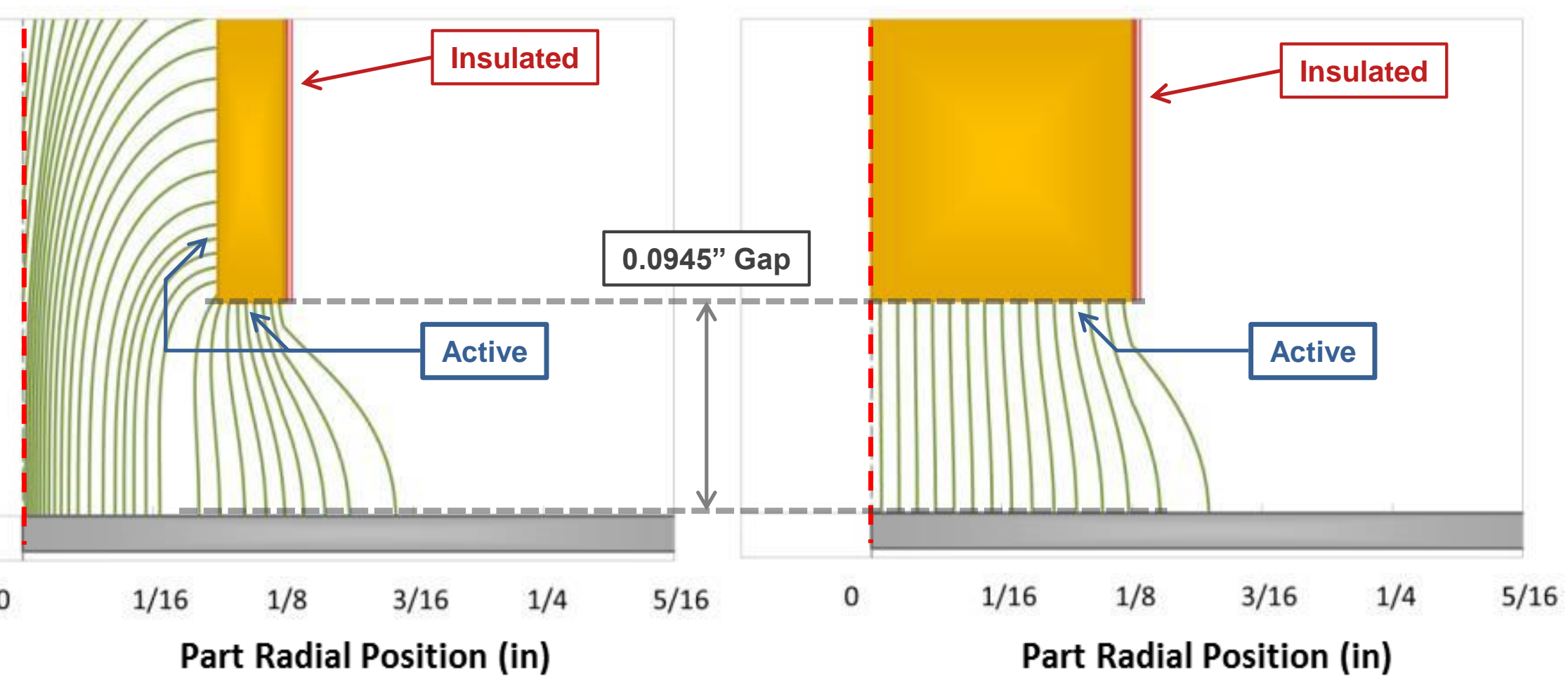

FARADAY תחתף TECHNOLOGY, INC. 


\section{Part Current Density Distribution - Rod vs Tube}

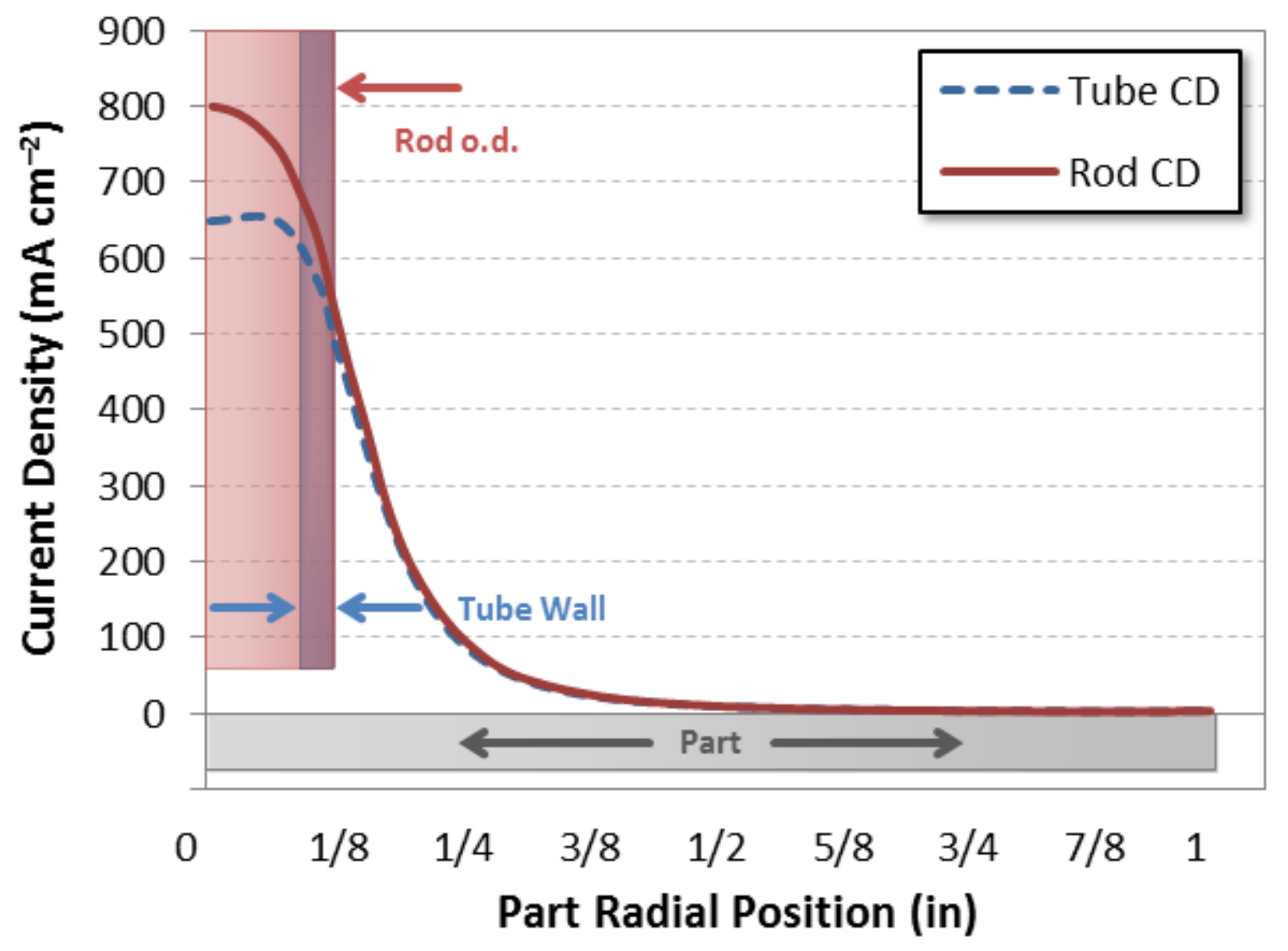

2V Applied Potential - $100 \mathrm{mS} / \mathrm{cm}$ Electrolyte Conductivity - 0.0945" Inter-Electrode Gap 


\section{Tool Shape Effect on Machined Profile}

- Time-dependent simulations WITH geometry deformation

- $2 \mathrm{~V}$ applied potential $100 \mathrm{mS} / \mathrm{cm}$ electrolyte conductivity 0.0945 " initial inter-electrode gap $0.00044 " / \mathrm{min}$ tool movement rate $1 / 32$ " walls for tube tools

$\mathbf{t}=\mathbf{0} \mathbf{h}$

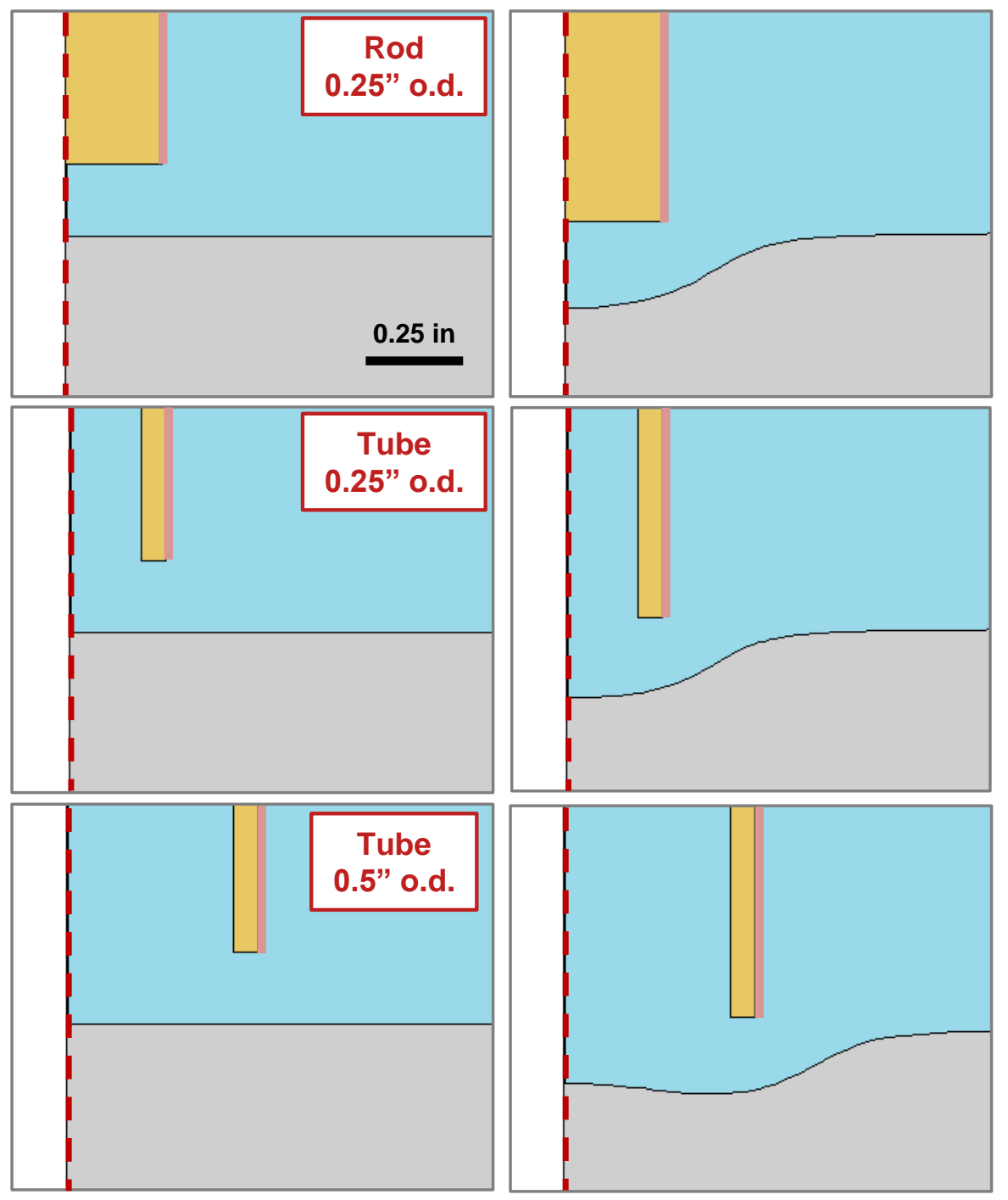




\section{Machining Fidelity - Inter-Electrode Gap}
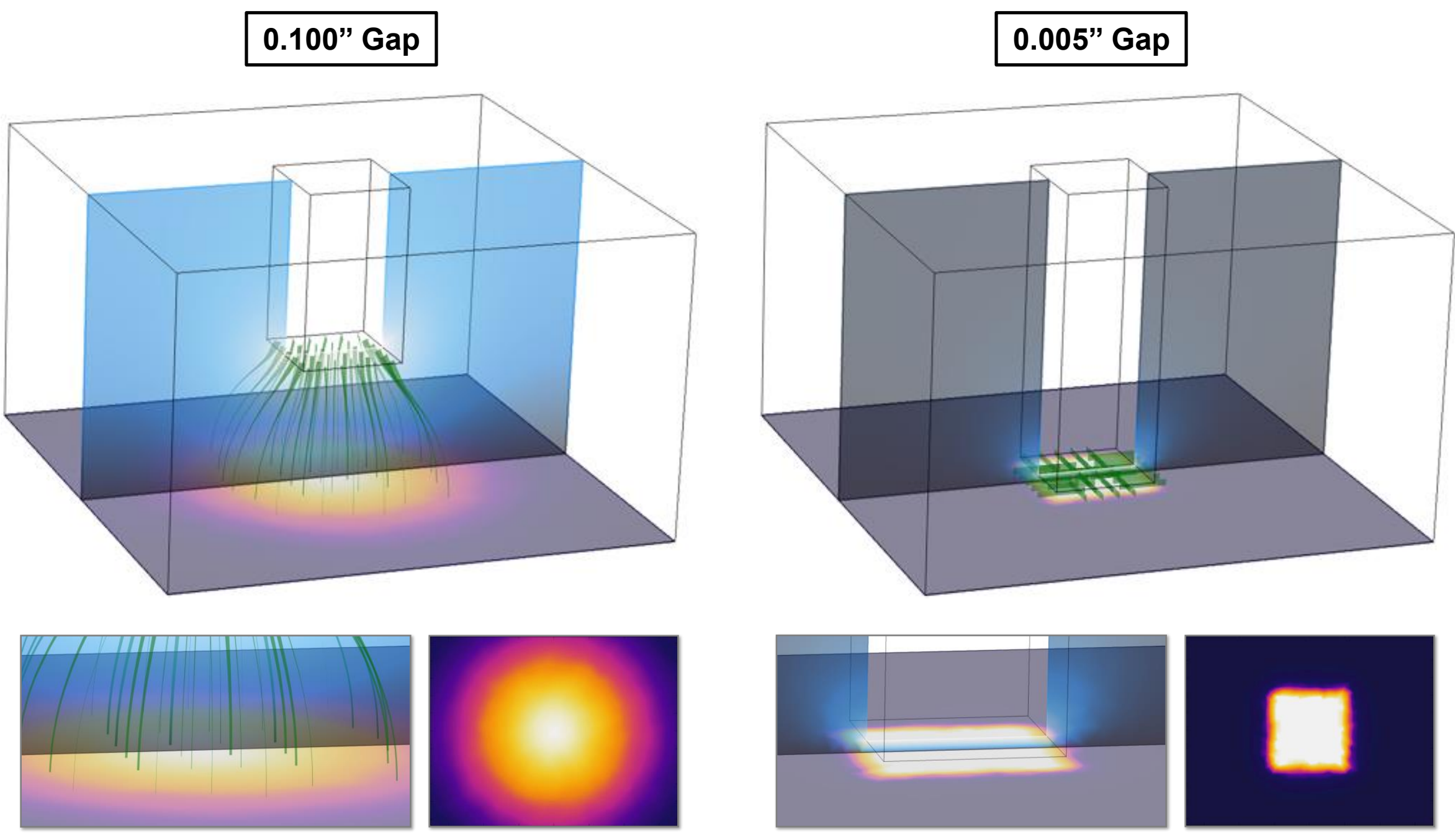

FARADAY תת ת ת TECHNOLOGY, INC. 


\section{FARADAY תЛתЛ,
TECHNOLOGY, INC.}

\section{ECM Tests}

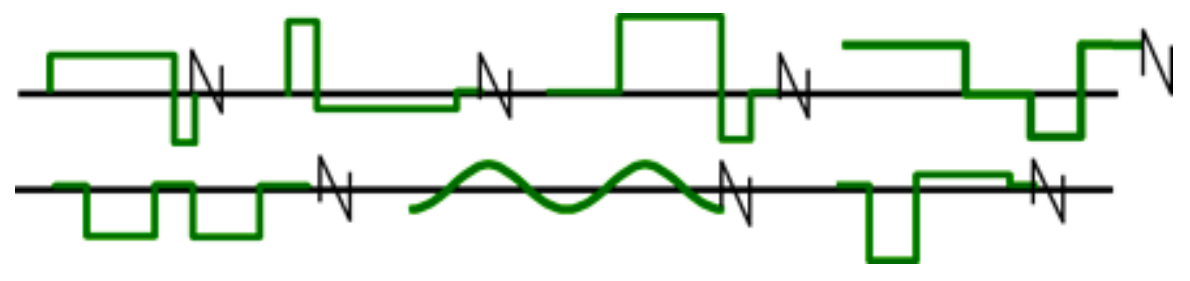




\section{ECM Apparatus}

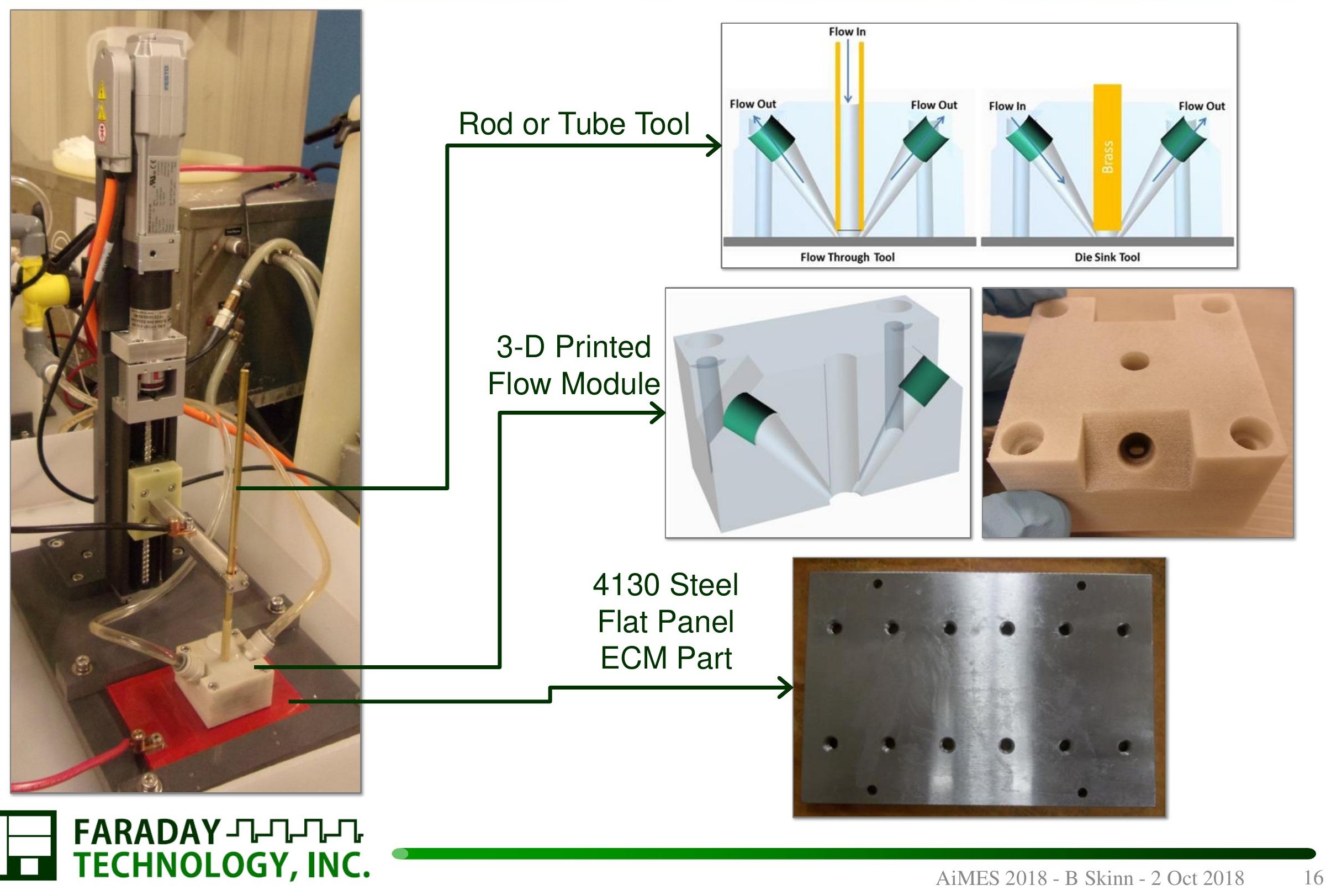




\section{ECM Experimental Parameters}

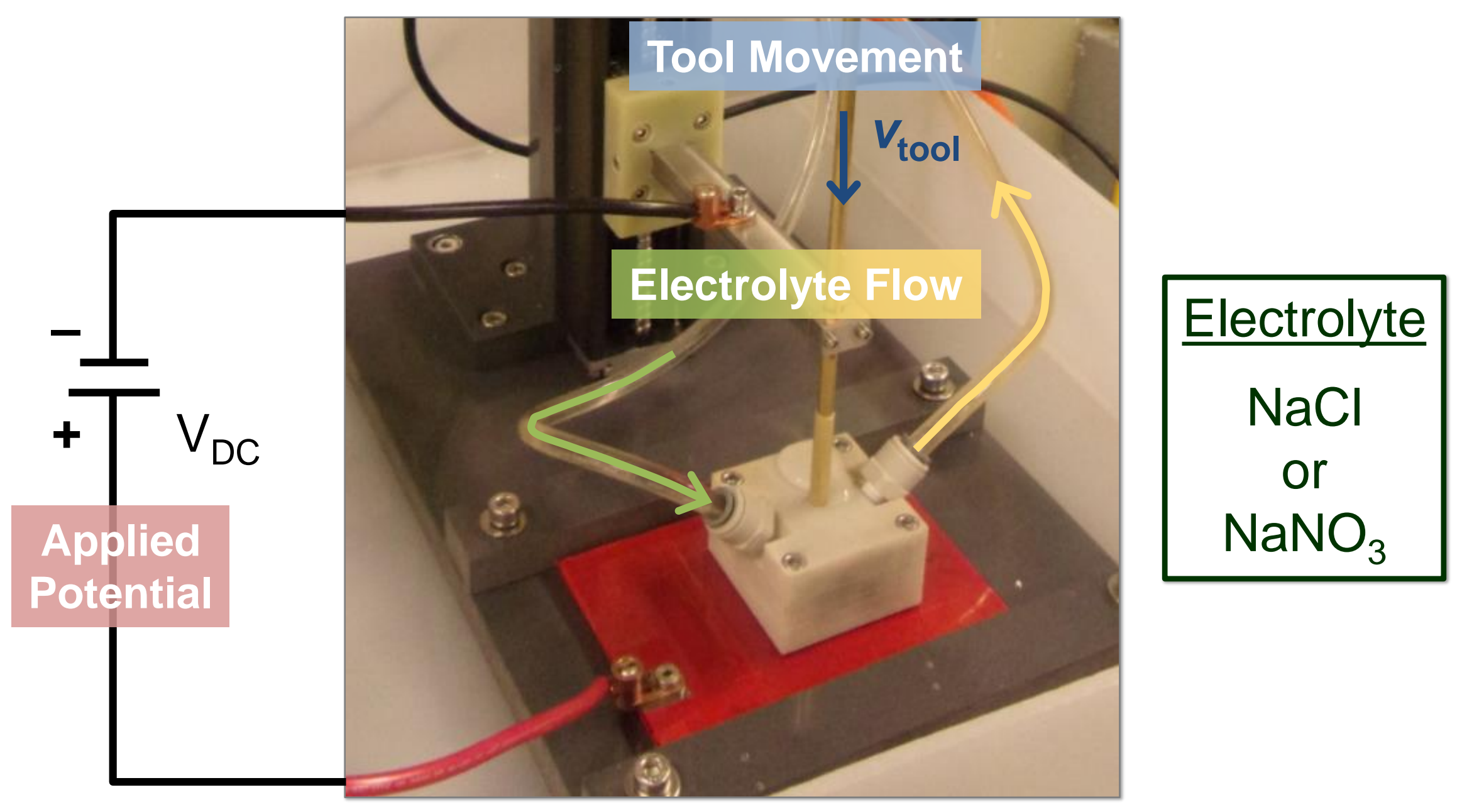

FARADAY ת ת TECHNOLOGY, INC. 


\section{Modeling - Rod/Tube Tool Tests}

Cross-Flow

Rod Tool

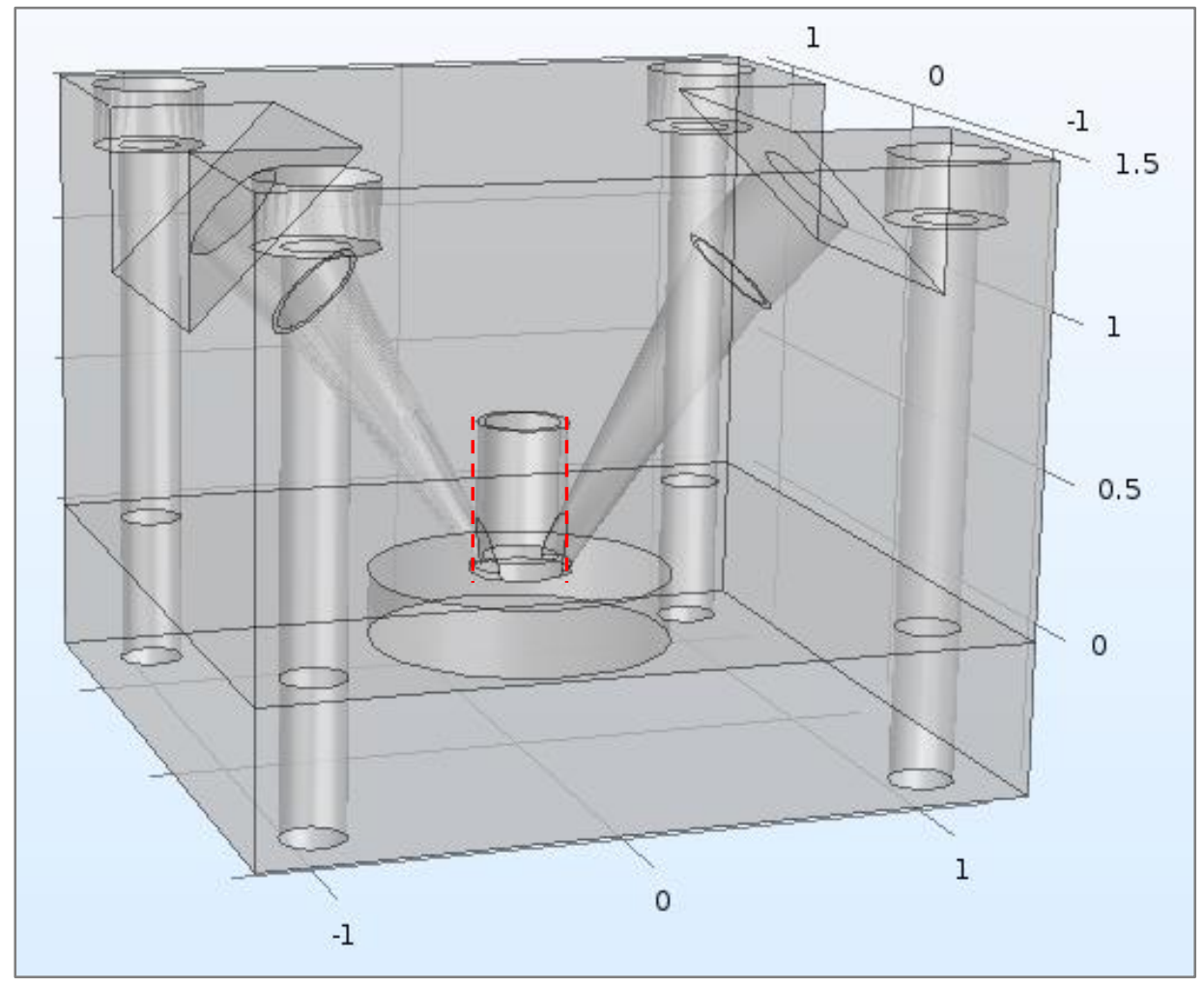

Flow-Through

Tube Tool

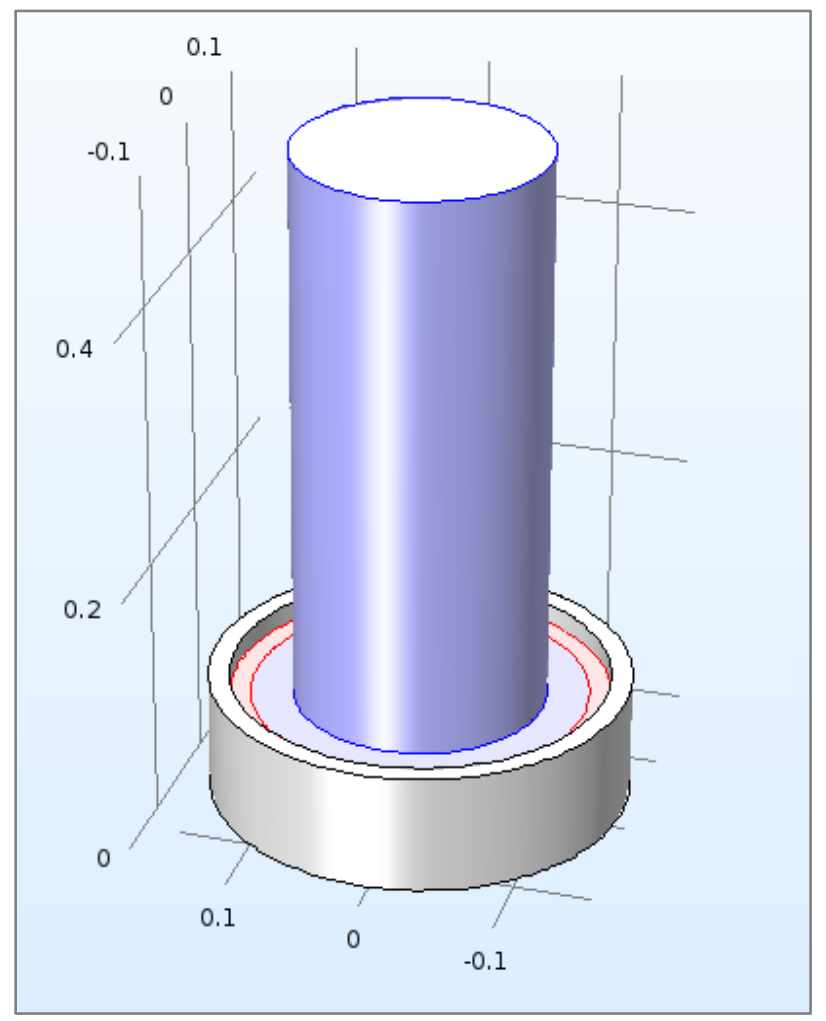

FARADAY תЛЛ TECHNOLOGY, INC. 


\section{Modeling - ECM Test Matrix - Rod/NaCl}

Rod Tool $-\mathrm{NaCl}-10 \mathrm{~V}_{\mathrm{DC}}-0.006^{\prime \prime} \mathrm{min}^{-1}-0.075^{\prime \prime}$ Initial Gap -20 min Duration
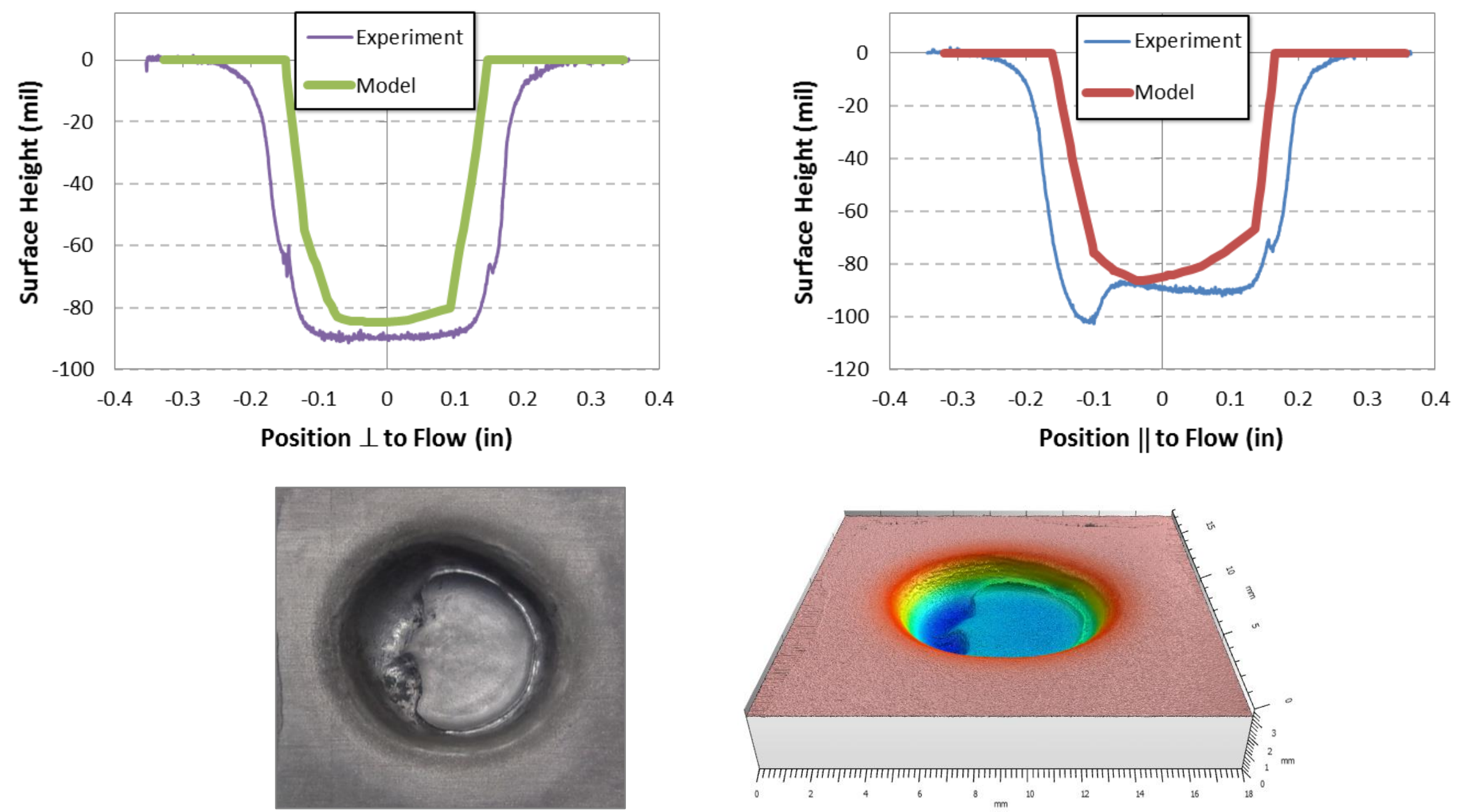

FARADAY תЛПЛ TECHNOLOGY, INC. 


\section{Modeling - ECM Test Matrix - Tube/NaCl}

Tube Tool $-\mathrm{NaCl}-10 \mathrm{~V}_{\mathrm{DC}}-0.006 " \mathrm{~min}^{-1}-0.075^{\prime \prime}$ Initial Gap -20 min Duration
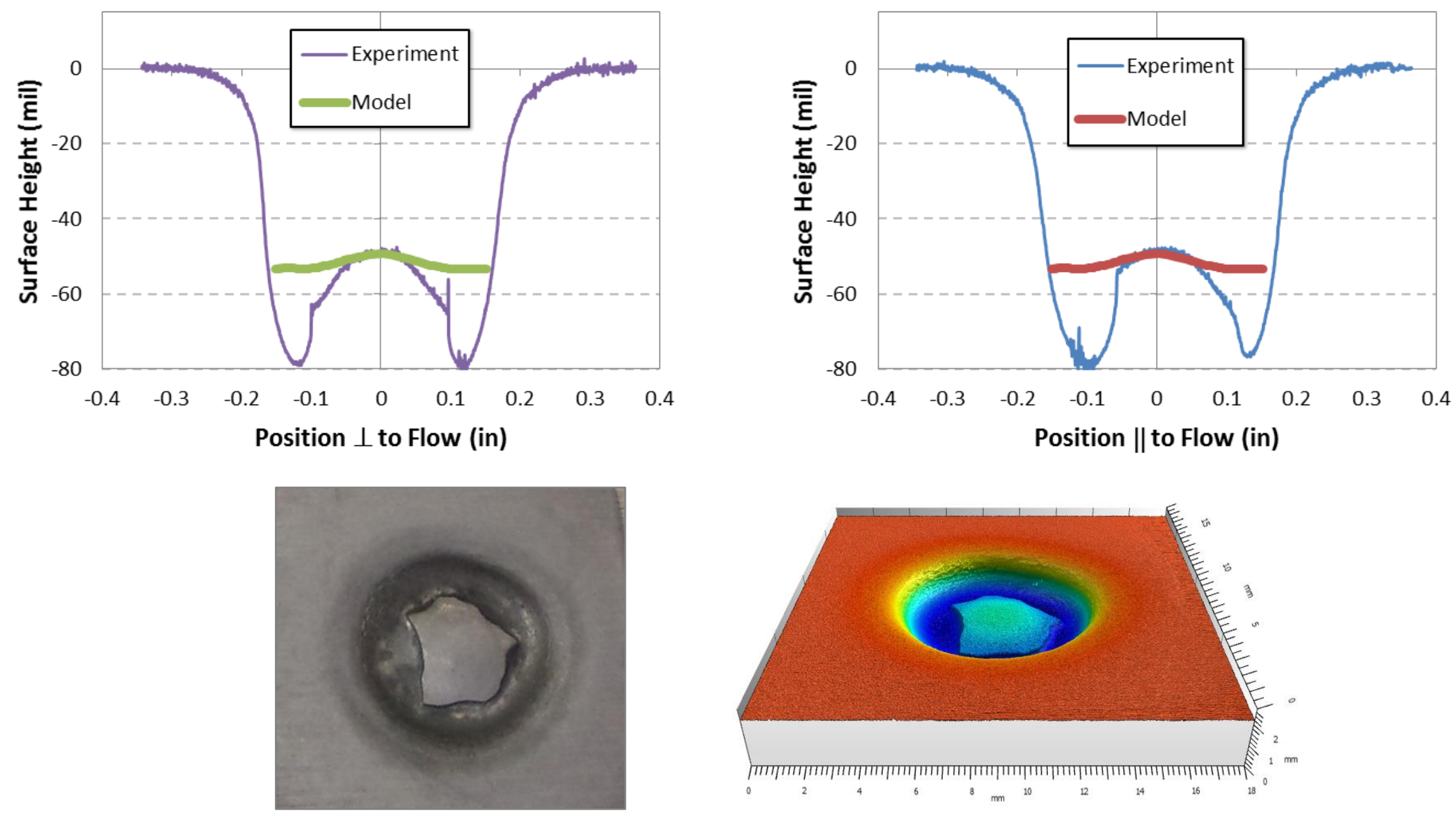

FARADAY תЛПЛ TECHNOLOGY, INC. 


\section{Summary}

- GOAL: Multiphysics modeling for rapid, accurate ECM tool design to obtain complex part shapes Reduce/eliminate multiple prototyping steps

- Preliminary modeling of core physics

- Primary current distribution

- Geometry deformation

- Small inter-electrode gap critical

- Additional physics likely needed for accurate simulations

- Subsequent modeling to include flow, surface reaction/polarization effects, etc.
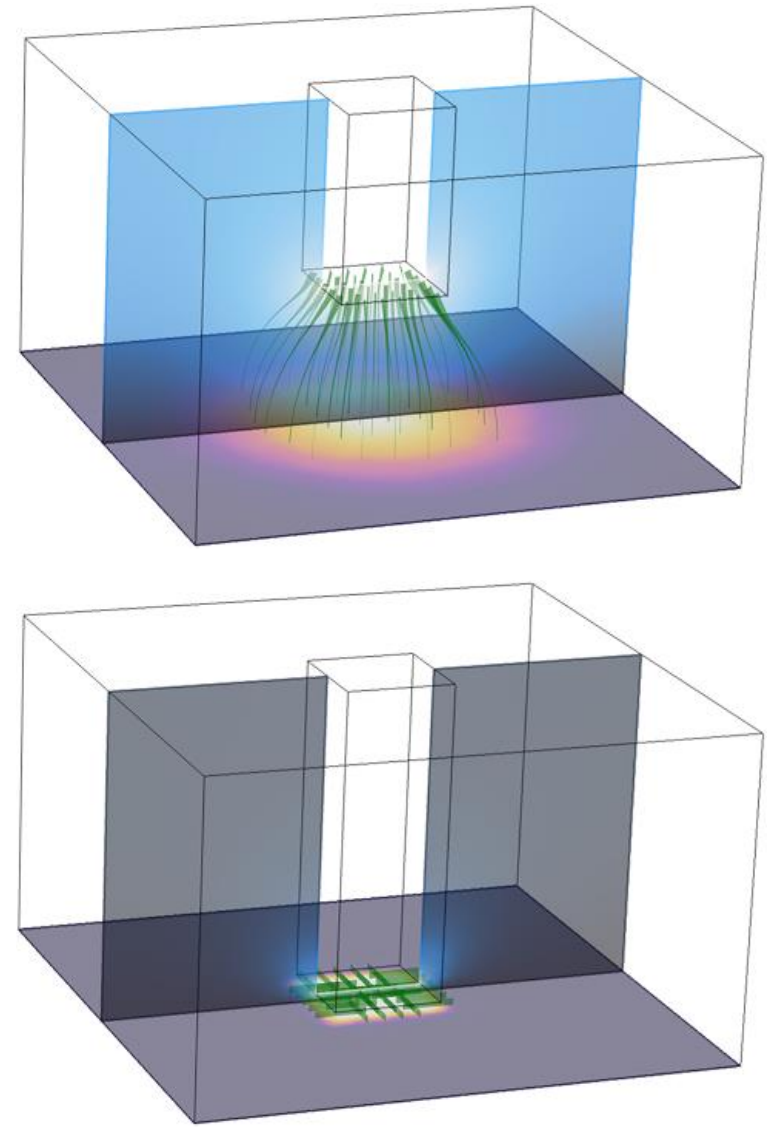


\section{Acknowledgment}

- This material is based upon work/resources supported by:

- Commercial Partners

- SBIR/STTR Grants

- US Army (synergistic activities)

- Faraday Corporate

- Contact Information:

Brian T. Skinn

Ph: +19378367749

Email: BrianSkinn@FaradayTechnology.com

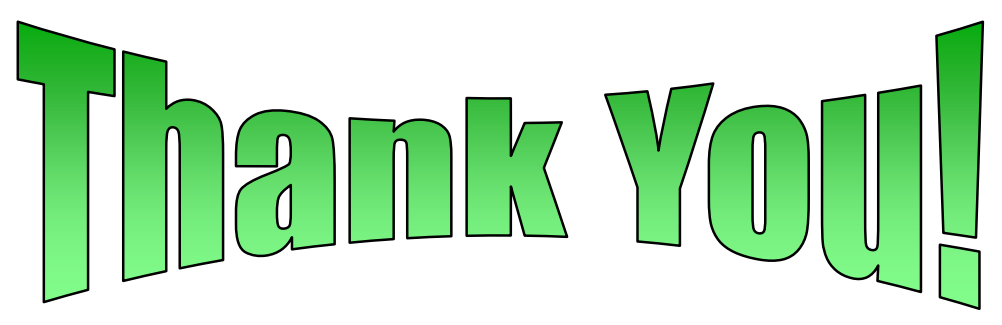

